\title{
The Study of Usefulness of Deep Breathing Exercise (Non Pharmacological Adjunct) on Blood Pressure in Hypertensive Patients
}

\author{
Dr.Vasuki $\mathrm{G}^{1}$, Dr.Sweety L.M ${ }^{2}$ \\ $\left({ }^{1,2}\right.$ Assistant Professor, Dept Of Physiology, Kanyakumari Govt Medical College, Asaripallam, Dr. MGR \\ Medical University, Chennai/ India )
}

\begin{abstract}
Background: Hypertension, the most common lifestyle disorder, is an important risk factor for cerebral and cardiovascular diseases. Stress relaxation techniques are considered a useful method for improving and maintaining both physical and emotional health. Deep breathing exercise helps in achieving efficient cardio respiratory functions with optimal sympathovagal balance which is an essential preventive strategy in the management of hypertension.
\end{abstract}

Aim and objective: To assess the effectiveness of deep breathing exercise on blood pressure and heart rate in patients with hypertension.

Materials and Methods: The study group comprised of 60 hypertensive subjects (age group 30-50 years) attending hypertensive clinic at Kanyakumari govt medical college hospital - control group comprised of 30 subjects and experimental group with 30 subjects. The experimental group subjects were taught to practice deep breathing exercise. The blood pressure and heart rate were recorded at 0,4,8,12 week intervals in both the experimental and control group.

Results analysis: Data was analysed using paired and unpaired 't' test. A highly significant decrease in blood pressure and heart rate was found in the experimental group.

Conclusion: Practising deep breathing exercise regularly shows advantages on autonomic and cardiovascular functions.

Keywords: Hypertension, Deep breathing exercise, Blood pressure and Heart rate.

\section{Introduction}

Systemic Arterial Hypertension, is a multifactorial disease which represents an independent, significant risk factor in the occurrence of cardiovascular diseases and cerebral vascular accidents. Among the risk factors for the development, maintenance and aggravation of hypertension, emotional stress is prominent. It is therefore necessary to emphasize stress management programs as a fundamental, non-pharmacological step in the management of hypertension.

Relaxation methods including yoga,meditation, and biofeedback have been demonstrated to reduce blood pressure in several studies. Deep and slow-paced diaphragmatic controlled breathing is common to many of these modalities and its use in lowering blood pressure has been reported in several studies, suggesting that reduction of respiratory rate lowers blood pressure by stimulating cardiovascular reflexes.

\section{Materials And Methods}

This study is a hospital based, cross sectional study. For the study group, 30 hypertensive subjects attending hypertensive clinic in Kanyakumari Govt Medical College Hospital and for the control group another 30 hypertensives are examined.

Inclusion criteria: Patients diagnosed with systemic hypertension, non smokers, non alcoholic; age group 30 to 50 years with systolic BP $>140$ and $<180 \mathrm{mmHg}$ or diastolic BP $>90$ and $<110 \mathrm{mmHg}$ or those using antihypertensive medication and $\mathrm{BP}<180 / 110 \mathrm{mmHg}$.

Exclusion criteria

Patients with

- Chronic respiratory disease.

- Chronic kidney disease.

- Coronary artery disease.

- Secondary hypertension.

- Malignant hypertension. 


\section{Study Protocol}

The protocol is explained to the participants and informed consent is obtained from each of them. By way of providing proforma, the required data is collected. They are advised to avoid consumption of tea / coffee 12 hours prior to reporting in the outpatient department. The participants are made to sit comfortably for 10 minutes. Height in meters, weight in kilograms are measured and BMI calculated. Then they are allowed to rest supine for fifteen minutes. Blood pressure is recorded using a sphygmomanometer following the standard technique.The same trained personnel recorded the blood pressure on all occasions. Initially baseline parameters like heart rate and blood pressure are recorded. Deep breathing training: Following this, participants in the experimental group are given training on deep breathing exercise (non pharmacological adjunct). Patients are instructed to perform the exercise twice a day for $10 \mathrm{~min}$ for four weeks and to come to out patient department after 4 weeks for the post data collection. During the study period, all the participants were instructed to continue with their existing level of physical activity and not to start or stop any other exercises. Reassessment of parameters- during their visit after 4 weeks, the heart rate and blood pressure are reassessed. The control group was excluded from deep breathing exercise training \& both the heart rate, blood pressure are reassessed during their follow up visit to the Hypertensive clinic. This procedure is repeated during subsequent visits in 8 and 12 weeks.

\section{Results Analysis}

Table - 1: Physical characteristics of subjects in control group \& experimental group

\begin{tabular}{|c|c|c|}
\hline Parameters & Control group & Experimental group \\
\hline Age (years) & $46 \pm 4.2$ & $47 \pm 4.2$ \\
\hline Height $(\mathrm{cms})$ & $159.8 \pm 3.6$ & $154 \pm 6.5$ \\
\hline Weight $(\mathrm{Kg})$ & $62.6 \pm 8.3$ & $68.3 \pm 4.4$ \\
\hline BMI & $25 \pm 4.3$ & $26 \pm 4.84$ \\
\hline
\end{tabular}

Table- 2: Comparison of blood pressure among the control group subjects at various weeks

\begin{tabular}{|l|l|l|l|l|}
\hline Parameters & $\begin{array}{c}\text { Initial Reading } \\
(\mathrm{mm} \mathrm{Hg})\end{array}$ & $\begin{array}{c}4 \text { week } \\
(\mathrm{mm} \mathrm{Hg})\end{array}$ & $\begin{array}{c}8 \text { week } \\
(\mathrm{mm} \mathrm{Hg})\end{array}$ & $\begin{array}{c}12 \text { week } \\
(\mathrm{mm} \mathrm{Hg})\end{array}$ \\
\hline $\begin{array}{l}\text { Systolic blood } \\
\text { pressure }\end{array}$ & $148 \pm 8.42$ & $146 \pm 10.1$ & $144.6 \pm 9$ & $143.8 \pm 7.8$ \\
\hline $\begin{array}{l}\text { Diastolic blood } \\
\text { pressure }\end{array}$ & $90 \pm 10.2$ & $91 \pm 8.2$ & $87.4 \pm 9.7$ & $87.5 \pm 8$ \\
\hline $\begin{array}{l}\text { Mean Arterial } \\
\text { Pressure }\end{array}$ & $109 \pm 8$ & $109.5 \pm 7$ & $106.5 \pm 7.5$ & $106.3 \pm 6.8$ \\
\hline
\end{tabular}

Table - 3: Comparison of blood pressure in the experimental group subjects before \& after exercise - at various weeks.

\begin{tabular}{|l|c|c|l|l|}
\hline Parameters & \multirow{2}{*}{$\begin{array}{c}\text { Initial reading } \\
(\mathrm{mm} \mathrm{Hg})\end{array}$} & \multicolumn{3}{|c|}{ After Exercise } \\
\cline { 3 - 5 } & 4 weeks $(\mathrm{mm} \mathrm{Hg})$ & 8 weeks $(\mathrm{mm} \mathrm{Hg})$ & $12 \mathrm{weeks}(\mathrm{mm} \mathrm{Hg})$ \\
\hline Systolic blood pressure & $139.1 \pm 14$ & $133.2 \pm 12^{* *}$ & $128.1 \pm 9.4^{* *}$ & $123.8 \pm 6.5^{* *}$ \\
\hline Diastolic blood pressure & $91.7 \pm 9.4$ & $88.1 \pm 9.5^{* *}$ & $83.9 \pm 7.6^{* *}$ & $80.3 \pm 4.4^{* *}$ \\
\hline Mean Arterial Pressure & $107.5 \pm 10.1$ & $103.1 \pm 9.4^{* *}$ & $98.6 \pm 7.3^{* *}$ & $94.8 \pm 4.5^{* *}$ \\
\hline
\end{tabular}

Table - 4: Comparison of parameters between control group \& experimental group at various weeks

\begin{tabular}{|c|c|c|c|c|c|c|}
\hline \multirow[t]{2}{*}{ Parameters } & \multicolumn{2}{|c|}{4 week $(\mathrm{mm} \mathrm{Hg})$} & \multicolumn{2}{|c|}{8 week $(\mathrm{mm} \mathrm{Hg})$} & \multicolumn{2}{|c|}{12 week $(\mathrm{mm} \mathrm{Hg})$} \\
\hline & Experimental & Control & Experimental & control & experimental & Control \\
\hline Systolic blood pressure & $133.2 \pm 12^{* *}$ & $146 \pm 10.1$ & $128.1 \pm 9.4^{* *}$ & $144.6 \pm 9$ & $123.8 \pm 6.5 * *$ & $143.8 \pm 7.8$ \\
\hline Diastolic blood pressure & $88.1 \pm 9.5 * *$ & $91 \pm 8.2$ & $83.9 \pm 7.6^{* *}$ & $87.4 \pm 9.7$ & $80.3 \pm 4.4^{* *}$ & $87.5 \pm 8$ \\
\hline Mean Arterial Pressure & $103.1 \pm 9.4^{* *}$ & $109.5 \pm 7$ & $98.6 \pm 7.3^{* *}$ & $106.5 \pm 7.5$ & $94.8 \pm 4.5^{* *}$ & $106.3 \pm 6.8$ \\
\hline
\end{tabular}

** denotes highly significant ' $\mathrm{P}$ ' value.

\section{Discussion}

In both the control and experimental groups, age and BMI of all subjects are comparable. In the control group, the mean age is $46 \pm 4.2$ years and mean BMI is $25 \pm 4.3 \mathrm{~kg} / \mathrm{m} 2$. In experimental group, the mean age is $47 \pm 4.2$ years and mean BMI is $26 \pm 4.84 \mathrm{~kg} / \mathrm{m} 2$. When the SBP, DBP \& MAP among the control group subjects at 4,8 and 12 week intervals are compared with their initial reading, it is not statistically significant. Comparing the SBP,DBP, MAP among the experimental group subjects, before and after exercise, at 4,8 and 12 week intervals it is found to be statistically significant. When comparing the parameters between control and experimental group at 4,8 and 12 week intervals, it is found to be statistically significant.

Various researches have proved that regular practice of slow deep and rhythmic breathing increases parasympathetic tone, decreases sympathetic activity, improves cardiovascular and respiratory functions, decreases the effect of stress and strain on the body thus improving physical and mental health. ${ }^{1,2}$ It increases baroreflex sensitivity and reduces chemoreflex activation, and reduces systolic, diastolic blood pressures as well 
as heart rate variations in hypertensive patients. ${ }^{3,4}$ The benefits of slow deep breathing have been observed in hypertensive individuals using stress control programs such as Lipp's Stress Control Training (Lipp, 1991, 2007) which demonstrated a reduction in the level of stress and blood pressure. Slow breathing has been shown to reduce the physiological parameters in stressful situations, as well as a reduction in anxiety and an improvement in cognitive performance. Various other studies have proved that the use of a multidisciplinary approach comprising clinical, pharmacological treatment and a program of intervention to control the risk factors, is more effective in reducing the rate of cardiovascular mortality and morbidity than treatment which is exclusively drug-based.

It is important to highlight that respiratory and cardiovascular systems share similar control mechanisms, thus alterations in one system will modify the functioning of the other. ${ }^{5}$ In essential hypertension, the sympathetic hyperactivity has been found to be associated with a generalized enhancement of the excitatory pathways, leading not only to sympathetic vasoconstriction, but also chemoreflex activation., Therefore, any modification in the respiratory control would also produce changes in the cardiovascular function. Tidal respiration is under voluntary control \& it is theoretically possible to induce such changes by voluntary modification of breathing. In turn, this practice brings about long-term benefits in cardiovascular and respiratory function.

In hypertensive subjects practicing slow deep breathing exercise at 6 cycles/min, the tidal volume increases to maintain minute ventilation. The reflex mechanism involves the pulmonary stretch receptors that are stimulated and afferents via vagi reaches nucleus tractus solitarious, reduces the chemoreflex sensitivity and thus might enhance the baroreflex, with an effect on reducing blood pressure and sympathetic activity as reported by Narkiewicz et al. ${ }^{8,9}$ The neural mechanism affecting the heart and peripheral vessels include impulses arising from brain stem respiratory centres. With slow deep breathing at 6 cycles/min, impulses from the respiratory center stop inhibiting the cardiovagal center and thus increases the vagal tone. This leads to slowing of heart rate and reduction in blood pressure as suggested by Pal G etal ${ }^{10}$ The effect of entraining all RR interval fluctuations causes them to merge at the rate of respiration and to increase greatly in amplitude. This increase in RR interval fluctuations has the effect of enhancing the baroreflex efficiency and lowering blood pressure. ${ }^{11}$

Spicuzza and colleagues suggested that long term practice of deep breathing independently reduces chemoreflex sensitivity. ${ }^{12}$ Adaptation of peripheral/ central chemoreceptor to chronic carbondioxide retention and adaptation of pulmonary stretch receptors to a habit of deep slow respiration may increase vagal afferent discharge to the brainstem nucleus tractus solitarius that sends projections to the thalamus and limbic system (Richard PB et al ). ${ }^{13}$

Subjects with essential hypertension have shallow respiration with an enhanced chemoreflex sensitivity. Slow deep breathing leads to a decrease in the central chemoreflex sensitivity which in turn depresses the vasomotor center. This reduces the sympathetic outflow, causing peripheral arteriolar vasodilatation, decreasing peripheral vascular resistance and thus reducing diastolic blood pressure. The decrease in sympathetic discharge decreases the tone of venules with increases in its capacitance, decrease in mean systemic filling pressure, decrease in venous return and finally reducing the systolic blood pressure.

\section{Conclusion}

Our study design is meant to highlight the potential benefits of slow deep breathing exercise in hypertensive patients. This study has proved that practicing slow deep breathing exercise 10 minutes twice a day for 12 weeks has significantly reduced the systolic and diastolic blood pressure. The efficacy, compliance and lack of side effect demonstrates that there is a potential benefit of using this in clinical practice as a nonpharmacologic therapy to achieve blood pressure control. ${ }^{14}$ It is also empasized that other strategies that complement deep breathing, such as changes in lifestyle - alteration in the food habits, physical exercise etc should be followed to control stress. ${ }^{15}$ This pilot study has to be taken to a larger group study, and also whether this breathing exercise is beneficial long term is an area for additional study. It is hoped that this would help to create awareness and the implementation of preventive strategies for hypertension and associated disorders.

\section{References}

[1]. Telles S, Nagarathna R, Nagendra HR. Breathing through 2. a particular nostril can alter metabolism and autonomic activities. Indian J Physiol Pharmacol 1994; $38: 133-7$.

[2]. Pramanik T, Sharma HO, Mishra S, Mishra A, Prajapati R, 4. Singh S. Immediate effect of slow pace bhastrika pranayama on blood pressure and heart rate. J Altern Complement Med 2009; $15: 293-5$.

[3]. Joseph CN, Porta C, Casucci G, Casiraghi N, Maffeis M, 5. Rossi M, et al. Slow breathing improves arterial baroreflex sensitivity and decreases blood pressure in essential hypertension. Hypertension 2005; $46: 714-8$.

[4]. Pinheiro CH, Medeiros RA, Pinheiro DG, Marinho Mde J. 6. Spontaneous respiratory modulation improves cardiovascular control in essential hypertension. Arq Bras Cardiol 2007; $88: 651-9$.

[5]. Bernardi L, Porta C, Spicuzza L, et al. Slow breathing increases arterial baroreflex sensitivity in patients with chronic heart failu re. Circulation. 2002;105:143-145. 
[6]. Dunlap ME. Cardiopulmonary baroreflexes. In: Izzo JL, Sica D, Black HR,eds. Hypertension Primer. 4th ed. Dallas, TX: American Heart Association; 2008:123-125.

[7]. Raupach T, Bahr F, Herrmann P, et al. Slow breathing reduces sympathoexcitation in COPD. Eur Respir J. 2008;32:387-392.

[8]. Francis DP, Ponikowski P, Coats AJ. Chemoreflex-baroreflex interactionss in cardiovascular disease. In: Bradley DT, Floras JS, eds. Sleep Apnea: Implications in Cardiovascular and Cerebrovascular Disease. New York, NY: Dekker; 2000:33-56.

[9]. Parati G, Izzo JLJr, Gavish B. Respiration and blood pressure. In: Izzo JL Jr, Sica D, Black HR, eds. Hypertension Primer. 4th ed. Dallas, TX: American Heart Association; 2008:136 -138.

[10]. Pal G, Velkumary S. Effect of short- term practice of breathing exercises on autonomic functions in normal human volunteers. IJMR 2004; 120:115- 121 .

[11]. Eckberg DL, Kifle YT, Roberts VL. Phase relationship between normal human respiration and baroreflex responsiveness. J Physiol. 1980;304:489- 502. Gokul R, Shillito L. Positive impact of yoga and pranayama on obesity, hypertension, blood sugar and cholesterol: A pilot assessment. J Altern complement Med 2007; 13: 1056-57.

[12]. Spicuzza L, Gabutti A, Porta C, Montano N, Bernardi L. Yoga and chemoreflex response to hypoxia and hypercapnia. Lancet 2000; 356: 1495-1496.

[13]. Richard PB, Patricia LG. Sudarsan kriya yogic breathing in the treatment of stress, anxiety and depression: Part I Neyrophysiologic model. The journal of alternative and complementary medicine 2005; 11: 189-201.

[14]. WHO and FAO 2003. Diet, nutrition and the prevention of chronic diseases: Report of a joint WHO/FAO Expert consultation. Report 916 Geneva: WHO 2003. [15]. Nagarajan S. Effect of slow breathing training for a month on blood pressure and heart rate variability in healthy subjects. Natl J Pysiol Pharmacol 2014; 4:245-248. 